\title{
Possible Relation between Lack of Posterior Vitreous Detachment and Severe Endogenous Endophthalmitis
}

\author{
Kazuhiko Umazume, Jun Suzuki, Yoshihiko Usui, \\ Yoshihiro Wakabayashi, and Hiroshi Goto \\ Department of Ophthalmology and Visual Sciences, Tokyo Medical University, Tokyo, Japan \\ Correspondence should be addressed to Kazuhiko Umazume; kazuhikouma@me.com
}

Received 29 July 2016; Accepted 7 September 2016

Academic Editor: Irini Chatziralli

Copyright (C) 2016 Kazuhiko Umazume et al. This is an open access article distributed under the Creative Commons Attribution License, which permits unrestricted use, distribution, and reproduction in any medium, provided the original work is properly cited.

Purpose. Endogenous endophthalmitis (EE) is a rare ocular disease caused by bacterial or fungal infection of intraocular spaces by hematogenous spread of pathogens from distant infectious loci in the body. We investigated the clinical characteristics and management of eyes with EE in ten consecutive patients. Methods. Ten patients (10 eyes) with EE treated at Tokyo Medical University Hospital in 2014 were reviewed. We retrospectively studied the causative organisms, systemic complications, pre/postoperative mean best-corrected visual acuity (BCVA), and status of posterior vitreous detachment (PVD). Results. The 10 patients comprised 8 males and 2 females, with mean age of 71.2 years. The causative organisms were bacteria in 6 eyes and fungi in 4 eyes. Systemic complications included septicemia or disseminated intravascular coagulation in 5 patients and diabetes mellitus in 4 patients. Postoperative BCVA was improved by $0.2 \log$ MAR or greater in 4 eyes and decreased in 4 eyes. Vitrectomy was performed in all eyes, and 4 required multiple surgeries. During vitrectomy, PVD was absent in 8 eyes, 4 of which showed retinal necrosis. The mean age of patients with no PVD was 71.2 years. Conclusion. Despite an advanced age, PVD was absent in the majority of patients with EE. PVD may be related to the pathogenesis and aggravation of EE.

\section{Introduction}

Metastatic endogenous endophthalmitis is a potentially sightthreatening intraocular infection resulting from hematogenous spread of microorganisms from a distant infective source within the body. The incidence of endogenous endophthalmitis is far lower than that of exogenous endophthalmitis including postoperative endophthalmitis, accounting for only 2 to $8 \%$ of all endophthalmitis cases $[1,2]$. The causative microorganisms are divided into bacteria and fungi, most commonly Candida species. Among bacteria, Grampositive bacteria are common in developing countries, while Gram-negative bacteria have been reported in East Asia region [3-5]. Compared to fungal infections, bacterial infections spread rapidly to intraocular tissues and require urgent management.

Basic local treatments for endogenous endophthalmitis include intravitreal injection of antibacterial or antifungal agents against the causative microorganisms as well as vitrectomy. In the case of phakic eyes, lens extraction is conducted simultaneously, and in the case of eyes with implanted intraocular lens, extraction of the intraocular lens should be considered as the situation demands. In addition, frequent instillation of ceftazidime and vancomycin eye drops after surgery is an accepted treatment for bacterial endophthalmitis $[6,7]$ and should be started as soon as possible after operation.

The predisposing risk factors for metastatic endogenous endophthalmitis include urinary tract infection, diabetes, HIV infection, intravenous hyperalimentation, liver abscess, and infectious endocarditis, while long-term hospitalization that has increased with the progression of population aging is also an important risk factor $[5,6,8,9]$. Furthermore, the association of posterior vitreous detachment (PVD) with retinal disease has attracted attention, accompanying the advent of optical coherence tomography $[10,11]$. The relationship 
between PVD and the pathogenesis or severity of endogenous endophthalmitis has not been reported. In the present study, we examined PVD pre- and intraoperatively in patients with endogenous endophthalmitis and correlated the presence of absence of PVD with clinical features.

Recently, the aging society has become an issue mainly in industrial countries, and the increase of endogenous endophthalmitis accompanying aging has been a concern. In this report, we describe the clinical characteristics, surgical methods, and outcomes of 10 cases of endogenous endophthalmitis treated in our department during the previous one year.

\section{Methods}

Ten consecutive patients (10 eyes; 4 left eyes and 6 right eyes) diagnosed with metastatic endogenous endophthalmitis at the Tokyo Medical University Hospital between April 2014 and March 2015 were studied. The patients comprised 8 males and 2 females, with mean age of $71.2 \pm 10.5$ years. All patients underwent 25-gauge vitrectomy using the Constellation Vision System (Alcon Laboratories Inc., Fort Worth). Ceftazidime and vancomycin were added to Balanced Salt Solution (BSS) to obtain final concentrations of $20 \mathrm{mg} / \mathrm{mL}$ and $1 \mathrm{mg} / \mathrm{mL}$, respectively, and used as intraocular irrigation solution. During surgery, vitreous fluid sample was collected for culture, smear, and microscopic examination to identify the causative microorganism. Blood culture was also performed before surgery.

The parameters analyzed were causative microorganism, clinical findings, pre- and postoperative visual acuity, surgical method, systemic disease, and PVD. Clinical findings focused on chemosis, keratic precipitates, fibrin deposition in anterior chamber, hypopyon, and fundus visibility. Posterior vitreous detachment was assessed by B mode ultrasonography before surgery and by microscopic observation during surgery.

Preoperative and postoperative visual acuity were compared by Student's $t$-test. Statistical analyses were performed using MedCalc version 12.1.1 (Mariakerke, Belgium). $P$ values less than 0.05 were considered significantly different.

\section{Results}

The causative microorganisms isolated from vitreous fluid samples collected during surgery and from blood cultures were bacteria in 6 eyes and fungi in 4 eyes. The bacterial species isolated were Klebsiella species in 2 eyes, Escherichia coli in 1 eye, Nocardia species in 1 eye, and unidentified Gramnegative bacteria in 2 eyes. The fungal species isolated were Candida species in 3 eyes and Cryptococcus in 1 eye (Table 1).

Preoperative clinical findings in all 10 eyes (Table 2) included chemosis in 7 eyes, keratic precipitates in 9 eyes, fibrin deposition in 8 eyes, hypopyon in 6 eyes, and invisible fundus in 5 eyes. Among the 4 eyes with fungal endophthalmitis, the fundus was visible in 3 eyes and difficult to observe in only 1 eye. Hence, fungal endophthalmitis appears to show slower progression of inflammation clinically compared to bacterial endophthalmitis.
TABLE 1: Cultures results.

\begin{tabular}{lc}
\hline Species & Number of eyes \\
\hline Bacteria & 2 \\
$\quad$ Klebsiella pneumonia & 1 \\
Escherichia coli & 1 \\
Nocardia & 2 \\
Gram-negative bacteria (detail unknown) & \\
Fungus & 3 \\
$\quad$ Candia albicans & 1 \\
Cryptococcus & \\
\hline
\end{tabular}

TABLE 2: Clinical features.

\begin{tabular}{lcc}
\hline Symptoms & Number of eyes & Number of eyes (fungal) \\
\hline Chemosis & 7 & 2 \\
Keratic precipitates & 9 & 4 \\
Fibrin & 8 & 3 \\
Hypopyon & 6 & 3 \\
Indistinct of fundus & 5 & 1 \\
\hline
\end{tabular}

When improvement in visual acuity was defined as gain of more than $0.2 \log$ MAR, postoperative visual acuity was improved in 4 eyes, unchanged in 2 eyes, and deteriorated in 4 eyes (Figure 1). In this analysis, the logMAR equivalent for counting fingers was 1.85 , hand motion was 2.3 , light perception was 2.8 , and no light perception was 2.9 [12]. Four eyes (40\%) were able to avoid becoming socially blind $(>0.7 \log$ MAR). Comparison of final visual acuity between bacterial endophthalmitis and fungal endophthalmitis showed that visual acuity tended to be slightly better in fungal endophthalmitis $(1.03 \pm 1.24)$ compared to bacterial endophthalmitis $(2.24 \pm 1.18)$, although there was no significant difference between two groups. Three eyes lost light perception after surgery. All three had bacterial endophthalmitis, caused by Klebsiella species, Escherichia coli, and Nocardia species in 1 eye each.

The risk factors in the 10 patients in the present study were diabetes in 4 patients, anastomotic leakage after colon cancer surgery in 2 patients, ruptured liver abscess in 1 patient, aspiration pneumonia in 1 patient, and long-term oral steroid therapy in 1 patient. Serious systemic complications consisted of septicemia in 1 patient and disseminated intravascular coagulation in 4 patients.

Regarding treatment method, all 10 eyes underwent vitrectomy, and 4 (40\%) required multiple surgeries before inflammation was resolved. In only one eye with severe pain and loss of light perception, eyeball enucleation was eventually conducted. In 2 eyes that had been implanted with intraocular lens before surgery, the intraocular lenses were extracted in both eyes.

Posterior vitreous detachment was absent in 8 eyes (80\%) before surgery. In these eyes, PVD was induced intentionally during surgery. In this series, a high frequency of the absence of PVD was observed (Figure 2). 


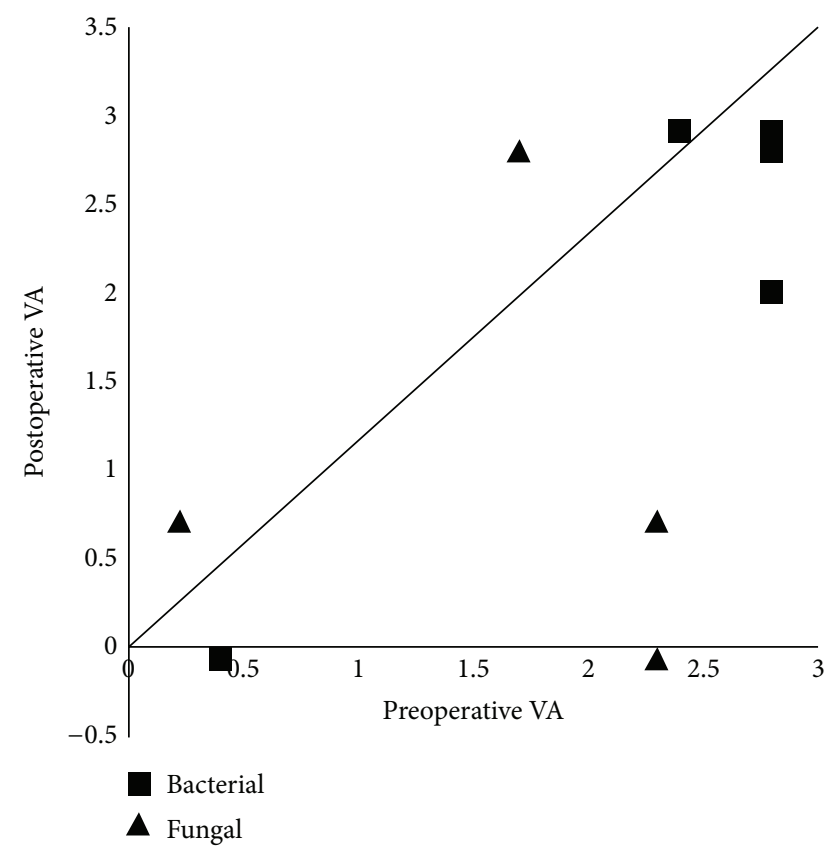

FIGURE 1: Comparison of pre- and postoperative visual acuity in 10 eyes treated for endogenous endophthalmitis. VA: visual acuity.

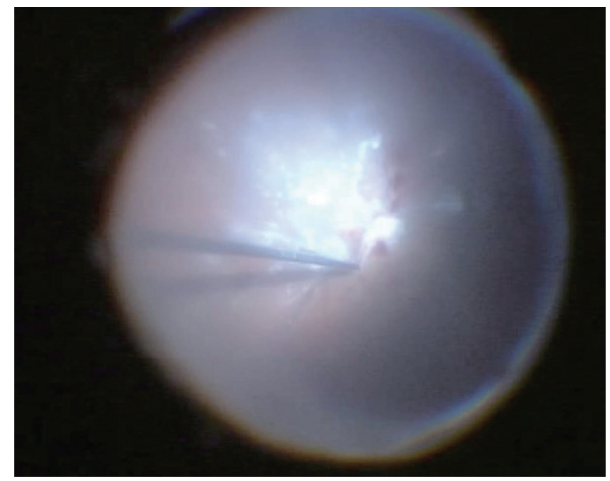

FIGURE 2: Fundus image shows induction of posterior vitreous detachment.

\section{Discussion}

According to an epidemiological survey of intraocular inflammations in Japan conducted in 2007, endogenous endophthalmitis accounted for 1 to $2 \%$ of intraocular inflammations [13]. Despite being a relatively rare intraocular disease, we observe a trend of increase at the Tokyo Medical University Hospital. While we treated a total of 13 cases between 2006 and 2013, we encountered 10 cases during one year in 2014. Regarding the age of disease onset, the patients with acute anterior uveitis $(\mathrm{m}=147)$, which is differential diagnosis of endogenous endophthalmitis, were $43.2 \pm 14.8$ years old. On the other hands, the patient with endogenous endophthalmitis in this series was $71.2 \pm 10.5$ years old. With the progression of population aging, the increase in endogenous endophthalmitis in the future is a concern.
The clinical picture of endogenous endophthalmitis is diverse. The misdiagnosis rates at presentation ranged from 16 to $63 \%[3,14]$. The early clinical symptoms include pain, hyperemia, floaters, and decreased vision. However, since some patients are not capable of complaining about the symptoms due to poor general condition, many patients are diagnosed in a serious state. In the present series, high rates of severe inflammatory findings were observed: fibrin deposition in 8 patients (80\%) and hypopyon in 6 patients $(60 \%)$. Furthermore, many patients with bacterial endophthalmitis had invisible fundus, probably as a result of rapid exacerbation of inflammation.

In the present study, all 10 eyes underwent vitrectomy. When inflammation spreads to the vitreous, prompt dissection of the vitreous is necessary. Although whether the causative organisms are bacteria or fungi can only be identified after performing culture of vitreous samples, we conducted surgery using an intraocular irrigation solution containing a mixture of vancomycin $(1 \mathrm{mg} / \mathrm{mL})$ and ceftazidime (20 $\mathrm{mg} / \mathrm{mL}$ ), as reported previously [15]. Although the vitreous body has to be dissected as much as possible, induction of an iatrogenic retinal break should be avoided, because this may affect retinal reattachment. In principle, we replaced the vitreous cavity with the intraocular irrigation solution containing antibiotics, but we used silicon oil when retinal tear was observed during surgery. In 4 patients (40\%), the first vitrectomy failed to resolve inflammation and multiple surgeries were required. In one patient with bacterial endophthalmitis, loss of light perception and uncontrolled pain eventually led to enucleation. Patients with endogenous endophthalmitis usually present with pain, and due to the high risk of expulsive hemorrhage during surgery, conducting surgery under general anesthesia is recommended if the general condition of the patient can tolerate anesthesia $[16,17]$.

The present study also examined the relation between PVD and endogenous endophthalmitis. Posterior vitreous detachment was not found before surgery in 8 eyes ( $80 \%)$, and PVD was induced during surgery in these eyes. The patients with no PVD had a mean age of $73.4 \pm 8.4$ years, which was older than the mean age of 54 to 57 years reported for the occurrence of PVD [18]. Furthermore, retinal necrosis is known to occur secondary to severe inflammation in endogenous endophthalmitis. In the present study, retinal necrosis was observed in 4 eyes (40\%), all of which had no PVD before surgery. These findings suggest that contact between the retina and the vitreous body may be associated with severe disease. A possible reason is that the gel-like vitreous body plays the role as a growth medium for the causative microorganisms. In vitro study has shown that the vitreous is associated with cell proliferation [19]; the same phenomenon may occur also in vivo. Currently, the use of ocriplasmin for vitreolysis has attracted interest [20,21], and use of this agent may also prevent aggravation of endogenous endophthalmitis.

Although the causative microorganisms have not changed compared to past reports, endogenous endophthalmitis remains a disease with poor visual outcome despite the advances in vitreous surgery. With the continuous progression of population aging, the number of cases is expected 
to increase in the future, and appropriate management including early diagnosis is required.

\section{Competing Interests}

The authors declare that there is no conflict of interests regarding the publication of this paper.

\section{References}

[1] S.-P. Chee and A. Jap, "Endogenous endophthalmitis," Current Opinion in Ophthalmology, vol. 12, no. 6, pp. 464-470, 2001.

[2] A. V. Rachitskaya, H. W. Flynn Jr., and J. L. Davis, "Endogenous endophthalmitis caused by Salmonella serotype B in an immunocompetent 12-year-old child," Archives of Ophthalmology, vol. 130, no. 6, pp. 802-804, 2012.

[3] T. L. Jackson, S. J. Eykyn, E. M. Graham, and M. R. Stanford, "Endogenous bacterial endophthalmitis: a 17-year prospective series and review of 267 reported cases," Survey of Ophthalmology, vol. 48, no. 4, pp. 403-423, 2003.

[4] V. Schiedler, I. U. Scott, H. W. Flynn Jr., J. L. Davis, M. S. Benz, and D. Miller, "Culture-proven endogenous endophthalmitis: clinical features and visual acuity outcomes," American Journal of Ophthalmology, vol. 137, no. 4, pp. 725-731, 2004.

[5] A. Lingappan, C. C. Wykoff, T. A. Albini et al., "Endogenous fungal endophthalmitis: causative organisms, management strategies, and visual acuity outcomes," American Journal of Ophthalmology, vol. 153, no. 1, pp. 162-e1, 2012.

[6] A. S. H. Tsai, S. Y. Lee, and A. H. E. Jap, "An unusual case of recurrent endogenous Klebsiella endophthalmitis," Eye, vol. 24, no. 10, pp. 1630-1631, 2010.

[7] M. Ang, A. Jap, and S.-P. Chee, "Prognostic factors and outcomes in endogenous Klebsiella pneumoniae endophthalmitis," American Journal of Ophthalmology, vol. 151, no. 2, pp. 338344.e2, 2011.

[8] V. Ho, L. Y. Ho, T. M. Ranchod, K. A. Drenser, G. A. Williams, and B. R. Garretson, "Endogenous methicillin-resistant Staphylococcus aureus endophthalmitis," Retina, vol. 31, no. 3, pp. 596601, 2011.

[9] J. Sridhar, H. W. Flynn Jr., A. E. Kuriyan, D. Miller, and T. Albini, "Endogenous fungal endophthalmitis: risk factors, clinical features, and treatment outcomes in mold and yeast infections," Journal of Ophthalmic Inflammation and Infection, vol. 3, no. 1, article 60, 2013.

[10] D. Gaucher, R. Tadayoni, A. Erginay, B. Haouchine, A. Gaudric, and P. Massin, "Optical coherence tomography assessment of the vitreoretinal relationship in diabetic macular edema," American Journal of Ophthalmology, vol. 139, no. 5, pp. 807-813, 2005.

[11] P. Carpineto and L. Mastropasqua, "Incomplete posterior vitreoschisis and vitreomacular traction," American Journal of Ophthalmology, vol. 140, no. 4, pp. 765-766, 2005.

[12] K. Schulze-Bonsel, N. Feltgen, H. Burau, L. Hansen, and M. Bach, "Visual acuities 'hand motion' and 'counting fingers' can be quantified with the freiburg visual acuity test," Investigative Ophthalmology \& Visual Science, vol. 47, no. 3, pp. 1236-1240, 2006.

[13] H. Goto, M. Mochizuki, K. Yamaki, S. Kotake, M. Usui, and S. Ohno, "Epidemiological survey of intraocular inflammation in Japan," Japanese Journal of Ophthalmology, vol. 51, no. 1, pp. 4144, 2007.
[14] M. I. Binder, J. Chua, P. K. Kaiser, G. W. Procop, and C. M. Isada, "Endogenous endophthalmitis an 18-year review of culturepositive cases at a tertiary care center," Medicine, vol. 82, no. 2, pp. 97-105, 2003.

[15] Y. Yonekawa, R. V. Paul Chan, A. K. Reddy, C. G. Pieroni, T. C. Lee, and S. Lee, "Early intravitreal treatment of endogenous bacterial endophthalmitis," Clinical and Experimental Ophthalmology, vol. 39, no. 8, pp. 771-778, 2011.

[16] Y.-Q. Zhang and W.-J. Wang, "Treatment outcomes after pars plana vitrectomy for endogenous endophthalmitis," Retina, vol. 25, no. 6, pp. 746-750, 2005.

[17] S. Jalali, N. Pehere, P. K. Rani et al., "Treatment outcomes and clinicomicrobiological characteristics of a protocol-based approach for neonatal endogenous endophthalmitis," European Journal of Ophthalmology, vol. 24, no. 3, pp. 424-436, 2014.

[18] J. Yonemoto, H. Ideta, K. Sasaki, S. Tanaka, A. Hirose, and C. Oka, "The age of oneset of posterior vitreous detachment," Graefe's Archive for Clinical and Experimental Ophthalmology, vol. 232, no. 2, pp. 67-70, 1994.

[19] D. Meitinger, D. M. Hunt, D.-T. Shih, J. C. Fox, and R. C. Hunt, "Vitreous-induced modulation of integrins in retinal pigment epithelial cells: effects of fibroblast growth factor-2," Experimental Eye Research, vol. 73, no. 5, pp. 681-692, 2001.

[20] J. A. Haller, P. Stalmans, M. S. Benz et al., "Efficacy of intravitreal ocriplasmin for treatment of vitreomacular adhesion: subgroup analyses from two randomized trials," Ophthalmology, vol. 122, no. 1, pp. 117-122, 2015.

[21] P. Stalmans and A. Girach, "Vitreous levels of active ocriplasmin following intravitreal injection: results of an ascending exposure trial," Investigative Ophthalmology and Visual Science, vol. 54, no. 10, pp. 6620-6627, 2013. 


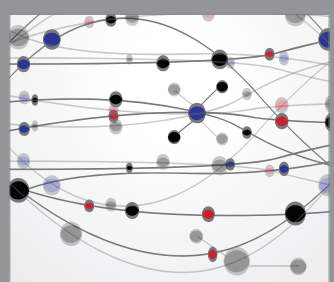

The Scientific World Journal
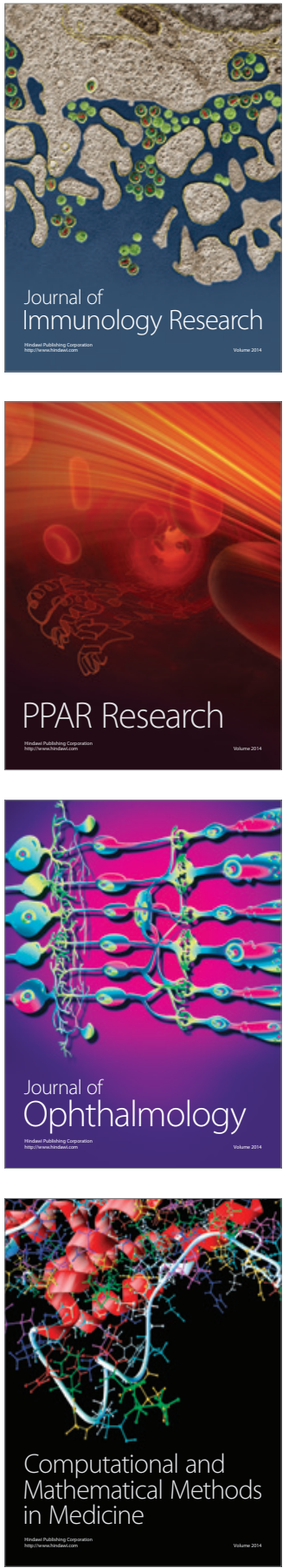

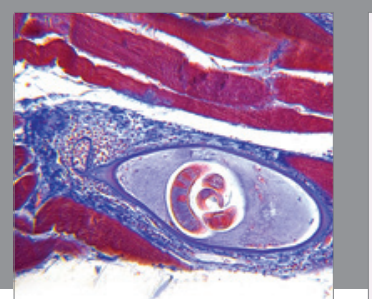

Gastroenterology Research and Practice

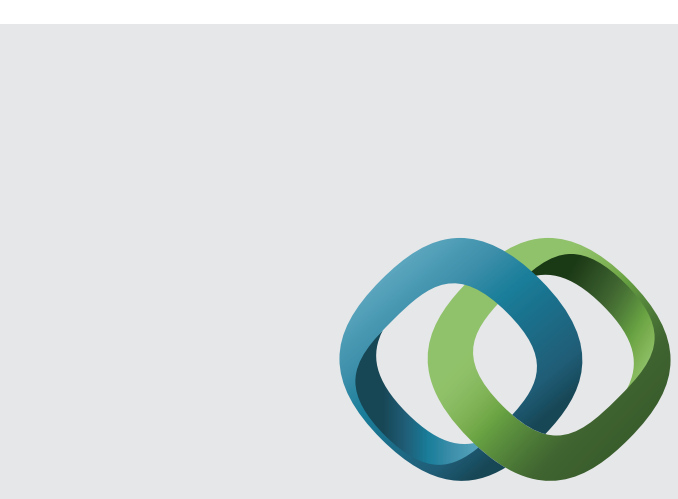

\section{Hindawi}

Submit your manuscripts at

http://www.hindawi.com
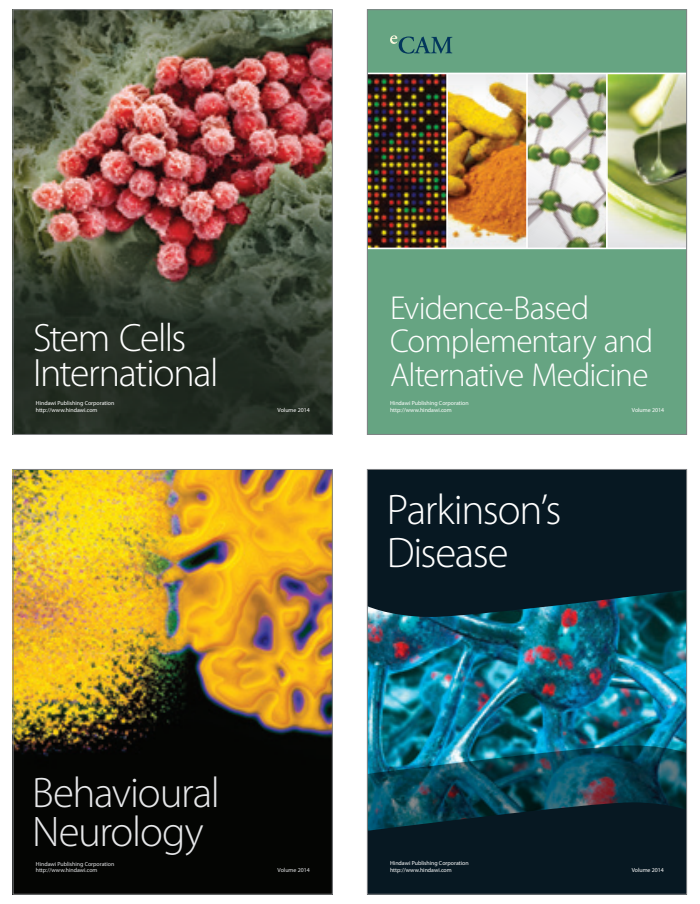
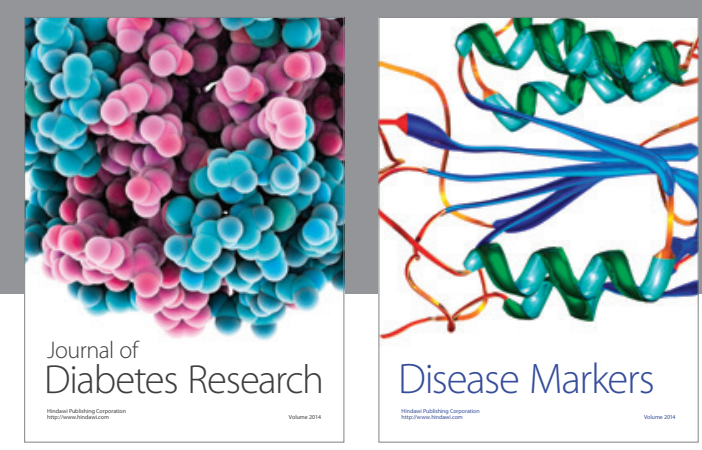

Disease Markers
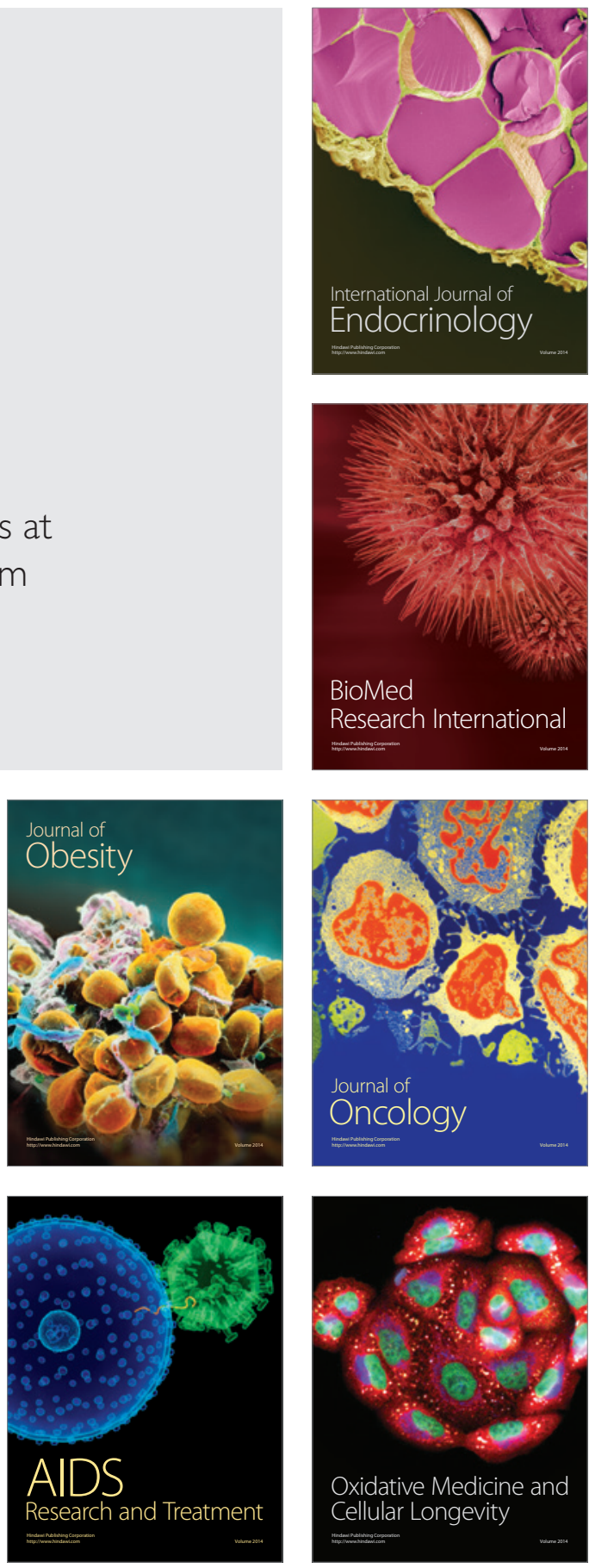